\title{
Incidental Presentation of Microfilaria in Cytological Smears at Different Sites with Coexisting Unusual Pathology
}

\author{
Ritu Sharma ${ }^{1 *}$, Abha Mishra ${ }^{1}$, Brig. Nikhilesh kumar ${ }^{1}$, Gaurav Kumar ${ }^{2}$ and H. S. Rawat ${ }^{3}$ \\ 'Dept. of Pathology, TSM Medical College, Lucknow, Uttar Pradesh, India \\ ${ }^{2}$ Dept. of E.N.T, TSM Medical College, Lucknow, Uttar Pradesh, India \\ ${ }^{3}$ Dept. of Surgery, TSM Medical College, Lucknow, Uttar Pradesh, India
}

\section{ABSTRACT}

Background: Microfilaria is a major public health problem in tropical and subtropical countries. Wuchereria bancrofti is the most common filarial infection. Despite its high incidence it is unusual to find microfilaria in fine needle aspiration cytology (FNAC) smear and body fluids. Out of these very few cases reported of coexisting microfilaria with neoplasm in the cytology. In literature however role of Microfilaria in tumorogenesis is controversial and not proven yet. The Aim of this study was showing importance of cytology (FNAC) in diagnosis of filariasis in lesions clinically anticipated to be of neoplastic /benign and to review the cytomorphology of bancroftian filaria and its association with neoplasm.

Methods: This is a retrospective study carried out in department of Pathology in T. S. Misra medical College and Hospital, Lucknow. Cytological records from last one year from December 2016 to November 2017 were taken of FNAC from different swelling, body fluids and screen for filariasis and its association with neoplasm.

Results: Out of 510 cases 17 cases showed microfilaria. Out of 17 cases 04 cases associated with malignant cells and remaining 13 cases with benign lesion.

Conclusion: Despite high incidence of filariasis, microfilaria in FNAC is not a very common finding. As a consequence, careful screening of cytological smear can be definitive diagnosis of early, asymptomatic and clinically unanticipated cases of bancroftian filariasis.

\section{Keywords: Microfilaria, Waucheria Bancrofti, FNAC.}

\section{Introduction}

Filaria, a vector-borne disease, is common in tropical countries like India. It is a major health problem in endemic areas, especially along sea coasts. There are three different filarial species that can cause lymphatic filariasis in humans. Most of the infections worldwide are caused by Wuchereria bancrofti out of all the species ${ }^{[1]}$. Though it is a common infection in India but it is very rare to find microfilaria in Fine Needle Aspiration Cytology (FNAC) smears and body effusions ${ }^{[2]}$. In our case, it was isolated from Thyroid gland, Breast, Metastatic lymph node, soft tissue swelling and various body fluids and urine. Hence, it has been reported for its rarity. The patient was treated with Diethylcarbamazine citrate (DEC) and there was a complete response to treatment, further confirming the diagnosis.

Bancroftian filariasis caused by Wucheria bancrofti. Man is the definite host, and mosquito is the intermediate host of Bancroftian and Brugian filariasis. The adult filarial worm lives in lymphatic vessels whereas microfilaria resides in peripheral blood. Filariasis may produce acute as well as chronic clinical manifestations or person may remain asymptomatic in endemic areas. However, in all the cases typical clinical manifestations of filariasis may not be seen. Pathological findings associated with filarial lesions are chronic inflammatory cell infiltrate consisting of lymphocytes, histiocytes, plasma cells, and eosinophils Association of filarial parasite with malignancy has been described but its role in tumorogenesis is not so far explained and it could be just a chance association.

Common methods of diagnosis of filariasis in this country are by demonstration of microfilaria in stained or unstained blood films, circulating filarial antigen detection and demonstration of organism in histopathological sections. Fluid cytology or fine needle aspiration cytology (FNAC) are rarely applied for routine diagnosis of clinically suspected filariasis. Filariasis has been reported in cytologic smears from various organs and sites like breast, soft tissue swelling, body fluids, salivary gland and axillary swelling ${ }^{[3]}$. 


\section{Materials and Method}

Cytological records of the year December 2016 to November 2017 in department of pathology of T.S. Misra medical college and Teaching Hospital were retrieved for diagnosed cases of filariasis. All the cases were clinically unsuspected of filariasis referred for FNAC by SURGICAL and ENT department. FNAC was taken from swelling different site and cytological smears were stained with Papanicolaou, H\&E and Giemsa stain. Slides with filarial organisms were reviewed and findings are tabulated. FNAC were done using $10 \mathrm{ml}$ syringe and 22 gauge needle under aseptic precautions. Aspirated material was spread on slides. In case of cystic lesions, cyto-centrifugation of fluid was carried out and repeats FNAC from the residual swelling was also carried out. Air dried smears were stained with Giemsa stain. Out of all these cases of FNAC, there were incidental findings of microfilaria in 17 cases.

\section{Results}

Total number of cytology during last one year was 510 that included FNAC from different sites, and body fluid cytology including urine cytology. Total numbers of cases with filariasis were only 17 (3.3\% of all cytological specimens) that include 12 cases of FNAC (2.3\% of 390 cases of FNAC) from different sites (Table-1) and 5 cases of body fluids ( $0.98 \%$ of 120 all fluids) pleural and ascitic fluid including urine cytology (Fig: 4). Out of these 17 cases of superficial swellings diagnosed as filariasis on FNAC, there were 14 cases in third and fourth decades of life. There were only three cases in 5th decade of life while no case was seen below the age of 20 years. Male and female were in 3:2 ratio (Fig: $1 \mathrm{~A}$ ) with age ranging from 20 years to 55 years. The records revealed that in none of the situation, the clinical diagnosis of filariasis was made by the clinicians. It was only after diagnosis on FNAC, treatment for the disease was started. In all the 17 cases microfilaria was identified as microfilaria of Waucheria bancrofti, based on its characteristic cytomorphology. Peripheral smears showed eosinophilia in five cases. Out of these five cases which showed eosinophilia (ranging from 10 to 30\%) (Table 3), in the smears from Inguinal swelling aspirate in one case showed both numerous coiled and single gravid female microfilariae (Fig: 3). Ova of the organism in addition to microfilaria were seen in two cases of FNAC, one from the soft tissue and other from inguinal lymph node. All the cases showed mixed inflammatory infiltrate composed of scattered lymphocytes, neutrophils and eosinophil. Four cases $(23.5 \%$ of 17 cases) were associated with malignancies (Fig: 1 B \& 2), (Table-2). Microfilaria load was more in the smears from nonneoplastic lesions in comparison to smears associated with malignancy.

Table1: Frequency of Filariasis in cytological smears.

\begin{tabular}{|c|c|c|c|c|}
\hline S.No & Specimen/FNAC & Total no. of cases & Number of cases with Filariasis & Percentages (\%) \\
\hline 1. & Lymph Nodes & 160 & 04 & 0.8 \\
\hline 2. & $\begin{array}{c}\text { Body fluids cytotology including } \\
\text { Urine cytology }\end{array}$ & 120 & 05 & 4.2 \\
\hline 3. & Thyroid Lump & 110 & 02 & 1.8 \\
\hline 4. & Soft Tissue & 60 & 04 & 6.7 \\
\hline 5. & Breast Lump & 40 & 01 & 2.5 \\
\hline 6. & Inguinal swelling & 20 & 01 & 5.0 \\
\hline TOTAL & & $\mathbf{5 1 0}$ & $\mathbf{1 7}$ & $\mathbf{3 . 3} \%$ \\
\hline
\end{tabular}

Table 2: Profile of patients with Filariasis associated with Malignancies.

\begin{tabular}{|c|c|c|c|c|}
\hline S.No & Age(yrs) & Sex & Site of specimen & Types of tumor \\
\hline 1. & 40 & F & FNAC Breast lump & Infiltrating duct Ca. \\
\hline 2. & 43 & M & Body fluid( Pleural) & Adenocarcinoma Metastatic \\
\hline 3. & 52 & $\mathrm{M}$ & FNAC Cervical lymph node & Adenocarcinoma \\
\hline 4. & 55 & $\mathrm{M}$ & FNAC Supra clavicular lymph node & Metastatic L.N. \\
\hline
\end{tabular}

Table 3: Profile of patients with Filariasis and associated peripheral blood findings.

\begin{tabular}{|l|l|l|l|l|}
\hline S NO. & Specimen/FNAC & No. of cases with filariasis & \multicolumn{2}{|c|}{ Associated PBS findings DLC TLC } \\
\hline 1. & Lymph node & 4 & $\begin{array}{l}\text { 3- Neutrophilia } \\
\text { 1-Eosinophilia }\end{array}$ & $\begin{array}{l}\text { 3- High count } \\
\text { 1-Normal count }\end{array}$ \\
\hline 2. & Body fluid cytology & 5 & $\begin{array}{l}\text { 3-Neutrophilia } \\
\text { 2-Eosinophilia }\end{array}$ & $\begin{array}{l}\text { 1-High count } \\
\text { 4-Normal count }\end{array}$ \\
\hline
\end{tabular}




\begin{tabular}{|l|l|l|l|l|}
\hline S NO. & Specimen/FNAC & No. of cases with filariasis & \multicolumn{2}{|l|}{ Associated PBS findings DLC TLC } \\
\hline 3. & Thyroid lump & 2 & 2-Neutrophilia & 2-Normal count \\
\hline 4. & Soft tissue & 4 & $\begin{array}{l}\text { 3-Neutrophilia } \\
\text { 1-Eosinophilia }\end{array}$ & $\begin{array}{l}\text { 2-High count } \\
\text { 2-Normal count }\end{array}$ \\
\hline 5. & Breast lump & 1 & 1-Eosinophilia & 1-Normal count \\
\hline 6. & Inguinal Swelling & 1 & 1-Netrophilia & 1-Normal count \\
\hline
\end{tabular}

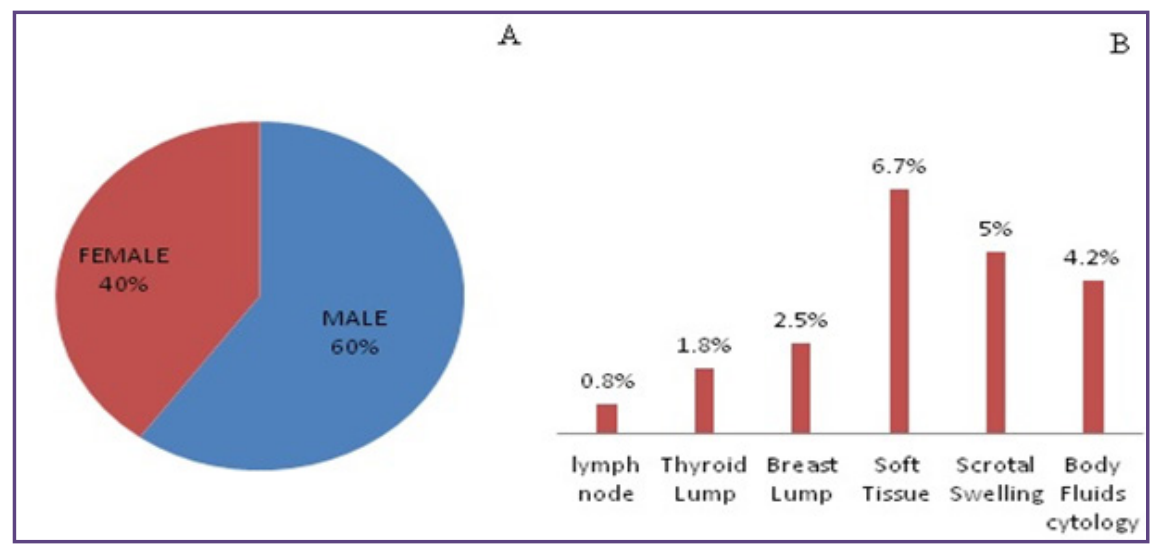

Fig. 1: A. Shows Male to Female ratio affected by Microfilaria, B. Shows Percentage of Microfilaria at different site.

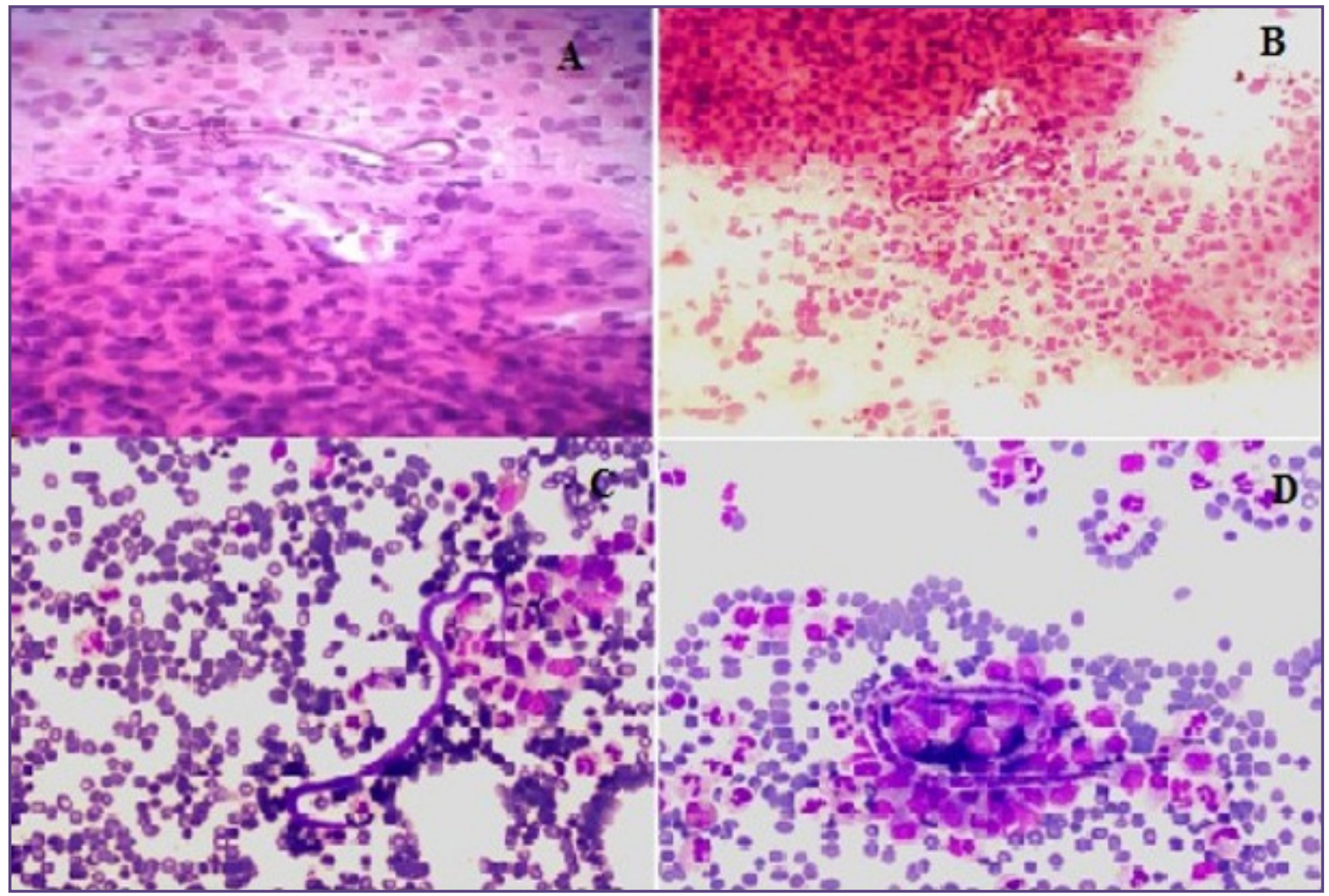

Fig. 2: A. Adult filarial worm with metastatic cluster of cells (H\&E 20x and 40x), B. FNAC smear from Supraclavicular lymph node, C \& D. FNAC smear from swelling cervical lymph node and soft tissue swelling of forearm. Shows adult Microfilaria with clusters of lymphoid cells. (MGG 40x). 


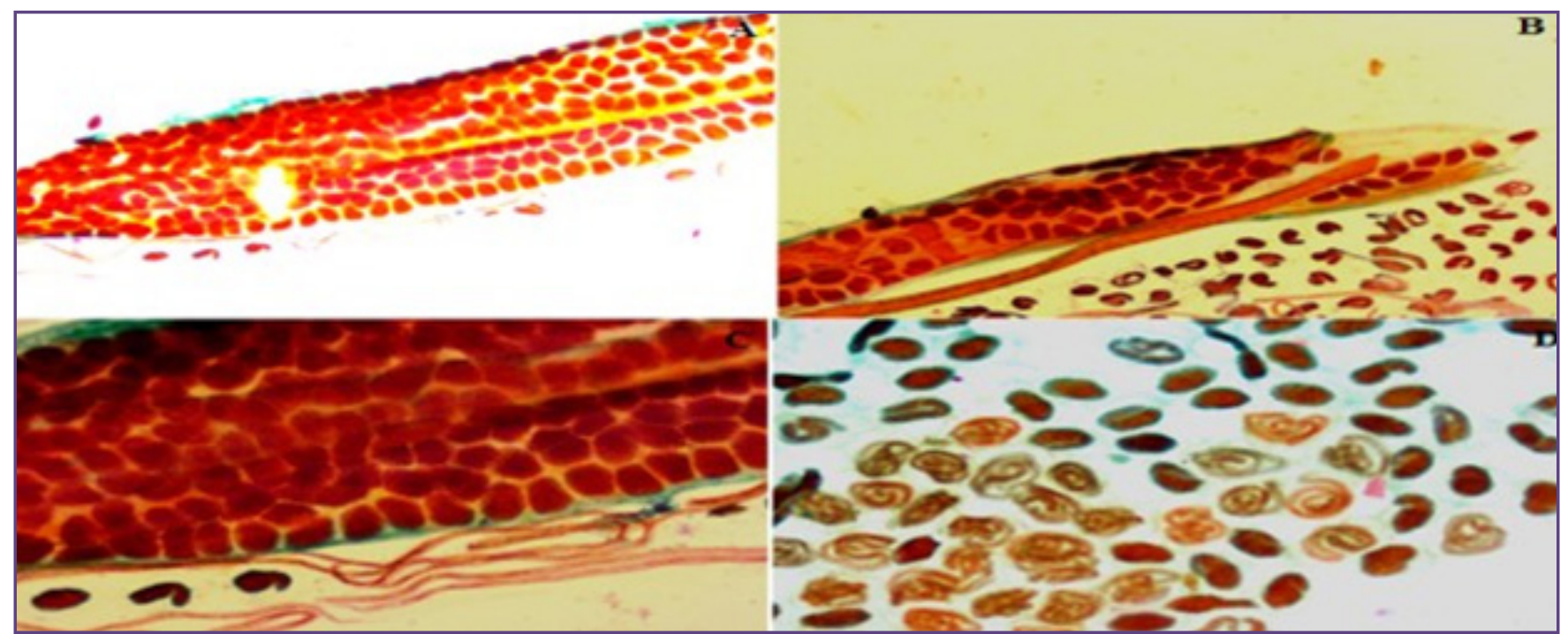

Fig. 3: A \& B. FNAC smear from swelling of thigh shows microfilaria in various stages- Gravid female, coiled stage and adult form (PAP Stained 20x and 40x), C \& D. FNAC smear of inguinal welling shows gravid female with coiled stage of microfilaria (PAP Stained 40x).

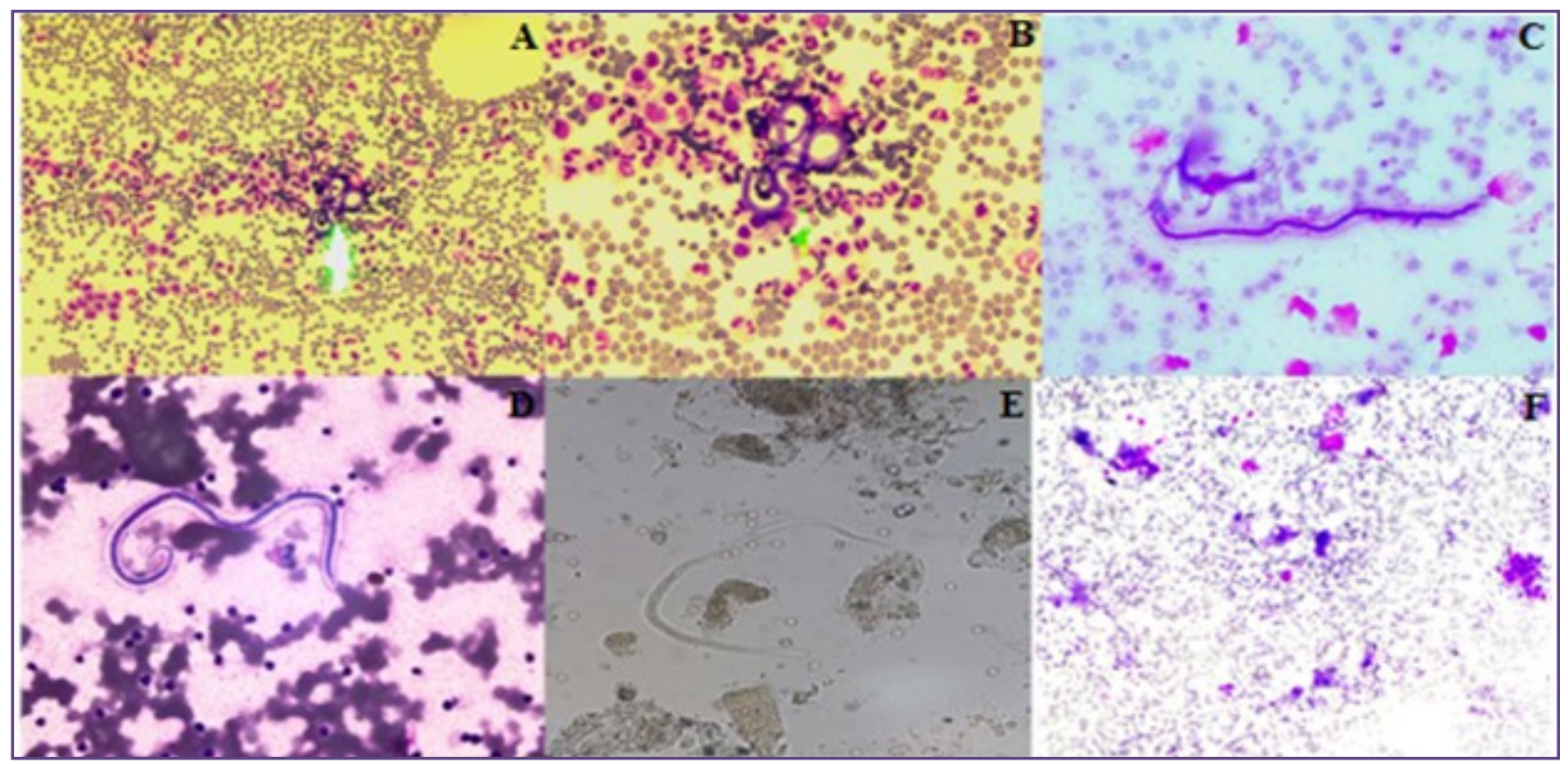

Fig. 4: A \& B. FNAC from breast lump shows Adult staged Microfilaria with inflammatory cells (MGG 20x and 40x). C, D \& E. Pleural fluid and wet mount smear of urine shows Adult microfilaria. (H\&E and MGG 40x), F. FNAC swelling breast lump shows adult microfilaria with infiltrating duct carcinoma (MGG 40X).

\section{Discussion}

Filariasis is a major public health problem in tropical countries, including India. It is caused by nematodes Wuchereria bancrofti, Brugia malayi, B. timori, Loa-loa, Onchocerca volvulus, Mansonella perstans, M.ozzardi ${ }^{[4]}$. Wuchereria bancrofti (90\%) and Brugia malayi (10\%) are the most common species causing filariasis in India ${ }^{[5,6,7]}$.
The disease caused by this parasite mainly involves lymph nodes, lymphatic system. It can also involve lungs, pleural, pericardial, ascitic fluid, ovarian cyst fluid, breast lump, bone marrow, bronchial aspirate, thyroid, parotid and gall bladder ${ }^{[6,8]}$. Many authors have reported microfilariae in breast lumps by FNAC smears ${ }^{[6,8,9]}$. Filarial parasite demonstrated in cytological smears from many unusual sites is an incidental finding ${ }^{[10,11]}$. 
The acute phase is characterized by fever, lymphangitis, lymphadenitis, epididymo-orchitis, and funniculitis. Headache, backache, muscle pain, insomnia, anorexia, urticarial rash, malaise, nausea and fatigue are common complaints. Eosinophilia and microfilaremia are common in acute phase. Chronic stage of bancroftian filariasis is characterized by lymphadenopathy, lymphedema, hydrocele, and elephantiasis. Adult worms live in the lymphatic channels of the definitive host and microfilaria is released and circulated in the peripheral blood. Female W. Bancrofti measures $80-100 \times 0.25 \mathrm{~mm}$ and the male $40 \times$ $0.1 \mathrm{~mm}^{[2]}$. The disease most frequently involves lymphatic. Other filariae mature in the skin and subcutaneous tissues, where they induce nodule formation and dermatitis. They were observed occasionally during routine cytological and histological examination from various benign lesions and they have rarely been detected in association with neoplastic lesions in cytological smears. In most of the reported cases microfilaria have been detected in lymph node, breast lump, bone marrow, pleural, ovarian cyst fluid and cervicovaginal smears.

In all 17 cases in the present study, microfilariae of $\mathrm{W}$. bancrofti were detected, as suggested by their typical morphologic appearance. The cephalic space at the anterior end is 5-7 microns long and the anterior nuclei are side by side. The caudal space at the pointed posterior end is 5-15 micron long and the terminal nuclei are elongated. Presence of ova and adult worms of filarial organism in cytological smears may or may not be associated with simultaneous presence of microfilaria.

Pleural fluid is uncommon site for microfilaria. Navaz et $a l^{[12]}$ stated that idiopathic pleural effusion must look for microfilaria, just like in our cases where patient have adenocarcinoma with pleural effusion. The presence of microfilaria along with neoplasm is generally regarded as a chance of association. In our cases also during routine FNAC of breast, lymph node along with infiltrating ductal carcinoma of breast (Fig 4A \& B) and adenocarcinoma and metastatic carcinoma of lymph node shows microfilaria in routine FNAC of the sites.

Microfilaria was a chance finding. This may be due to its transmigration along with metastatic emboli. It has also been suggested that such aberrant migration to these dead end sites is probably determined by local factors such as lymphatic blockage by scars or tumors and damage to the vessel wall by inflammation, trauma or stasis. Rich blood supply in the tumors could be a reason for concentration of parasites at these $\operatorname{sites}^{[13]}$. Gupta has emphasized that microfilariae wander in tissue fluid and may get entrapped in needle during aspiration ${ }^{[14]}$.
In present study none of the cases were clinically anticipated of having filarial infection. In most of the reported cases filariasis was diagnosed in cytology in clinical unsuspected cases. Despite high incidence of filariasis, microfilaria in FNAC is not a very common finding. As a consequence, careful screening of cytological smear can be definitive diagnosis of early, asymptomatic and clinically unanticipated cases of bancroftian filariasis, especially with the amicrofilaremic state.

\section{Conclusion}

India is endemic for filarial infection, it is necessary to examine carefully FNAC and body fluid smears from different body swelling at different unsuspected locations for microfilaria. FNAC is a rapid, simple procedure and is diagnostic tool for the diagnosis of microfilaria.

It is interesting to notice that there are very few reported cases of filarial organisms associated with tumors in histological sections as compared to cases reported in cytological specimens as is the case in present study. Microfilariae have been detected in association with metastatic malignant cells in pleural fluid, peritoneal fluid ${ }^{2}$ and pericardial fluid. In our study four case of microfilaria was detected in association with metastatic cluster of cell at different lymph node and pleural fluid. Their presence along with benign and malignant tumor is controversial issue and needs to be explored further. Their presence in association with tumors of lymph nodes and lymphatic can be explained as they are normal habitation for the filarial organism, however, this view does not completely explain their association with tumors from other sites.

\section{References}

1. Mondal SK. Incidental detection of filarial in fine needle aspirates: A cytologic study of 14 clinically unsuspected cases at different sites. Diagn Cytopathol. 2012;40:292-6. http://dx.doi.org/10.1002/dc.21557

2. Yenkeshwar PN, Kumbhalkar DT, Bobhate SK. Microfilariae in fine needle aspirate: A report of 22 cases. Indian J Pathology \& Microbiology. 2006;49:365-69. [PubMed]

3. Chakrabarti N, Konar K, Mala, Bandyopadhyay A, Bose K. Incidental Detection of Microfilaria in Cytology: Report of three Cases. Ann Clin Pathol. 2014;2(4):1033.

4. Nutman Thomas B, Weller Peter F. Filarial and related Infections: Harrison's Principles of Internal Medicine. 16th Edition. Vol. 1. McGraw Hill; pp. 1260-63.

5. Park K. Lymphatic Filariasis: Park's textbook of preventing and social medicine. 22nd Edition.Jabalpur: Banarasidas Bhanot; 2013. pp. 245-50.

6. Varghese R, Raghuveer CV, Pai MR, Bansal R. Microfilariae in cytologic smears: A report of six cases. ActaCytol. 1996;40:299-301. 
7. Muddamwar VG., Rane MS., Shewale SP., Sameer MA., Deshpande M. S., Phasge G. Midarm lymphadenopathy A rare presentation of filariasis in a 20 year old female in Nanded Region. International Journal of Pharmaceutical Science Invention. May 2014; 3(5):12-16

8. Pantola C., Kala S., Agarwal A., Khan L. Microfilaria in cytological smears at rare sites coexisting with unusual pathology: A series of seven cases. Trop. Parasitol. 2012;2(1):61-63

9. Mitra SK, Mishra RK, Verma P. Cytological diagnosis of microfilariae in filariasis endemic areas of eastern Uttar Pradesh.J Cytol. 2009;26(1):11-14.

10. Reddy GS, Balasundaram S. Filarial pericardial effusion. J Indian Med Assoc. 1977;68:125-6.
11. Andola S, Naik A. Microfilaria and filarial granulomas from fine niddleaspirates . A study of 25 cases. Southeast Asian J Trop Med Public Health . 2011; 42(6): 1351-1358.

12. Alam K. Navaz, Madhusudan P. Raikar, Vishak Acharya, and Sanmath K. Shetty. Pleural effusion: An unusual cause and association. Lung India.2013;2(30):158-160.

13. Katti TV, Athanikar VS, Ananthrao AS, Rathod CV. Cytodiagnosis of microfilarial lymphadenitis coexistent with metastatic squamous cell carcinoma in a left cervical lymph node: An unusual presentation. Ann Nigerian Med 2012;6:47-9.

14. Gupta S, Gupta R, Bansal B, Singh S, Gupta K, Kudesia M. Significance of incidental detection of filariasis on aspiration smears: a case series. Diagn Cytopathol 2010;38(7):517-20.

*Corresponding author:

Dr. Ritu Sharma, H.No. 1404, HIG, Sector- I, Lda Colony, Kanpur Road, Lucknow, Uttar Pradesh, India, PIN - 226012.

Phone: +91 5229559815295

Email: drritzsharma@gmail.com

Financial or other Competing Interests: None. 